\title{
Multivariate non-normally distributed random variables in climate research - introduction to the copula approach
}

\author{
C. Schölzel ${ }^{1,2}$ and P. Friederichs ${ }^{2}$ \\ ${ }^{1}$ Laboratoire des Sciences du Climat et l'Environnement (LSCE), Gif-sur-Yvette, France \\ ${ }^{2}$ Meteorological Institute at the University of Bonn, Germany
}

Received: 28 November 2007 - Revised: 28 August 2008 - Accepted: 28 August 2008 - Published: 21 October 2008

\begin{abstract}
Probability distributions of multivariate random variables are generally more complex compared to their univariate counterparts which is due to a possible nonlinear dependence between the random variables. One approach to this problem is the use of copulas, which have become popular over recent years, especially in fields like econometrics, finance, risk management, or insurance.

Since this newly emerging field includes various practices, a controversial discussion, and vast field of literature, it is difficult to get an overview. The aim of this paper is therefore to provide an brief overview of copulas for application in meteorology and climate research. We examine the advantages and disadvantages compared to alternative approaches like e.g. mixture models, summarize the current problem of goodness-of-fit (GOF) tests for copulas, and discuss the connection with multivariate extremes. An application to station data shows the simplicity and the capabilities as well as the limitations of this approach. Observations of daily precipitation and temperature are fitted to a bivariate model and demonstrate, that copulas are valuable complement to the commonly used methods.
\end{abstract}

\section{Introduction}

In climate research there is a natural interest in estimating multivariate probability density functions. The motivation is obvious. The climate system can be defined as the coupled system of atmosphere, ocean, cryosphere, biosphere, and lithosphere. This system as well as each subsystem has to be regarded as stochastic, even if the data to be analysed originate from a deterministic numerical model. This is based on the fact that the climate system is a high-dimensional and nonlinear system. Detailed considerations on the stochastic

Correspondence to: C. Schölzel

(schoelzy@gmx.net) nature can be found in e.g. Lorenz (1964); Eckmann and Ruelle (1985). The variables involved are therefore treated as random variables and described in terms of probability distributions or probability density functions.

Taking a closer look at the state of the art, it can be seen that estimating multivariate probability density functions is mostly limited to the multivariate normal distribution or mixtures of it. The reason is, that there are numerous kinds of univariate probability distributions, but only in a few cases there is a native multivariate analogue. Textbooks in statistics, especially concerning those with application to climate research (Storch and Zwiers, 1999; Wilks, 2005), often introduce the multivariate normal distribution, but do not provide alternatives. Finding native multivariate distribution, analogously to the univariate distributions, is a non-trivial problem.

It needs not to be discussed, that meteorological or climatological data often turn out to be non-Gaussian, e.g. precipitation, wind speed, cloud cover, or relative humidity, which belong to bounded or skewed distributions. So, the essential question is how to deal with random variables, that do not belong to a multivariate normal distribution, for example the joint distribution of several precipitation measurements. How can we describe a random vector that has e.g. normally as well as gamma distributed marginals? Figures 1 and 2 show examples as discussed below.

The following section gives a brief overview on how the problem has been addressed so far. Section 3 introduces the copula concept, while Sect. 4 considers details on different kinds of copulas, their estimation, and goodness-of-fit tests. Since multivariate extreme value theory and copulas are related, Sect. 6 focuses on this problem separately. Section 7 shows examples of how to apply the copula approach to typical meteorological parameters. Section 8 briefly summarizes strengths and weaknesses of the copula approach with respect to alternatives. 


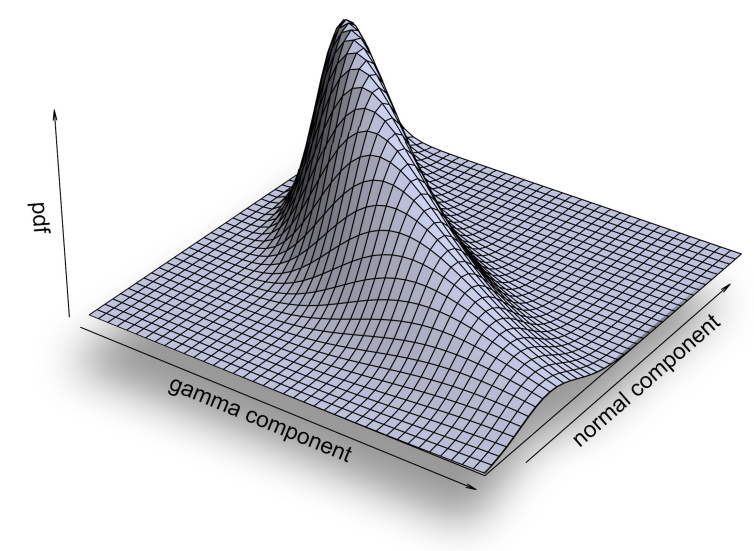

Fig. 1. Example of a bivariate probability density function for a random vector with normally and Gamma distributed vector components. The dependence among the components is and given by a normal copula with correlation -0.85 .

\section{Background and related work}

Before answering the question how multivariate non-normal probability density functions can be estimated, we should briefly summarize why this is a non-trivial problem. Assume a multivariate random variable with different marginal distributions. While the parametric description of the marginal distributions is mostly not a problem because of the various kinds of univariate distributions, the dependencies between non-normally distributed components cause some trouble. It is difficult to find a general definition of the correlation between the different random vector components (Cario and Nelson, 1997), so that there is no natural or unique way to extend the marginal distributions to a true multivariate distribution. So, how has this problem been addressed so far?

A well known way to circumvent this problem is the use of mixture models, e.g. multivariate Gaussian mixture models (Marin et al., 2005; McLachlan and Peel, 2000). While they are more flexible with respect to the covariance structure than the approaches presented below, they have two problems. On the one hand, they become quite complex in higher dimensions. On the other hand, they do not account for the original marginal distribution of the random vector components. This is an important aspect, which becomes even more relevant in context of extreme value theory.

Other parametric descriptions of the relationship among the variables are also either complex, e.g. Bézier distribution (Wagner and Wilson, 1994), which leads to a high number of parameters, especially in higher dimensions, or are restricted in modeling marginals and covariance structure, e.g. the Johnson distribution (Johnson, 1949). Within this context, multivariate extensions of the lognormal or gamma distribution are not an option, either, since all components would have to follow the same kind of marginal distribution. Nevertheless, the Johnson distribution (Johnson, 1949), later summarized by (Wilson, 1997), was an early solution to describe multivariate non-normal populations and is also the precursor of the approach presented in the following.

In the field of decision and risk analysis, the classical approach to modeling uncertainty is to write the joint distribution of the random variables as the product of marginal and conditional distributions (Clemen and Reilly, 1999). A practical problem of this approach is that the complexity of the estimation process grows exponentially with the number of random variables. Therefore, the use of so called copulas (e.g. Nelsen, 2006) became more and more popular over the last decade. Aiming at assessing risk, copulas are widely used in finance studies and related fields (e.g. Breymann et al., 2003; Haas, 1999), and more recently in hydrology (Renard and Lang, 2007; Genest et al., 2007; Bardossy and $\mathrm{Li}, 2008)$.

Although this is not done without critics (see Sect. 8), it seems to be a feasible method to estimating multivariate nonnormally distributed random variables for many real world problems. It is shown below, that together with the marginal distributions the copula concept is simply a mathematical reformulation of a multivariate probability distribution or density function, which is possible for (almost) every kind of multivariate distribution. The concept is straight forward and equivalent, but despite its simplicity and its success in other fields, it is less known in climate research and meteorology. Only a few applications are known (e.g. Michele and Salvadori, 2003; Vannitsem, 2007; Vrac et al., 2005).

\section{Multivariate random variables and copulas}

We assume a $m$-dimensional random vector $\boldsymbol{X}$ with marginal cumulative distribution functions $F_{X_{1}}, \ldots, F_{X_{m}}$ with domain R, i.e. nondecreasing and $F_{X_{1}}(-\infty)=0$ and $F_{X_{1}}(\infty)=1$. Sklar's theorem (1959) then says that the joint distribution $F_{X}$ of this random vector can be written as a function of its marginal distributions,

$F_{\boldsymbol{X}}(\boldsymbol{x})=C_{\boldsymbol{X}}\left(F_{X_{1}}\left(x_{1}\right), \ldots, F_{X_{m}}\left(x_{m}\right)\right)$

where $C_{X}:[0,1] \times \cdots \times[0,1] \rightarrow[0,1]$ is a joint distribution function of the transformed random variables $U_{j}=F_{X_{j}}\left(X_{j}\right)$ for $j=1, \ldots, m$. Due to this transformation the $U_{j}$ always have uniform marginal distributions. Moreover, if marginal distributions are continuous, the copula function is unique (Nelsen, 2006). For simplicity, we assume continuous and differentiable distribution functions $F_{X_{1}}, \ldots, F_{X_{m}}$, although this approach can be easily extended to a mixture of discrete and continuous random variables. In this case, $C_{\boldsymbol{X}}$ is unique 
and can also be expressed by

$C_{X}\left(u_{1}, \ldots, u_{m}\right)=\int_{0}^{u_{1}} \ldots \int_{0}^{u_{m}} c_{X}\left(u_{1}^{\prime}, \ldots, u_{m}^{\prime}\right) d u_{1}^{\prime} \ldots u_{m}^{\prime}$

with $u_{j}=F_{X_{j}}\left(x_{j}\right)$. The function $C_{X}$ is called a copula and $c_{X}$ the corresponding copula density. Sometimes the copula density is called dependence function, because it encodes the dependencies between the random variables.

The important consequence of Sklar's theorem is that every joint probability density can be written as the product of the marginal probability densities and the copula density.

$f_{\boldsymbol{X}}(\boldsymbol{x})=f_{X_{1}}\left(x_{1}\right) \cdots f_{X_{m}}\left(x_{m}\right) \cdot c_{\boldsymbol{X}}\left(u_{1}, \ldots, u_{m}\right)$

Hence it is equal to one for independent random variables, which is consistent with basic probability rules. Bivariate examples are given in Figs. 1 and 2. Nevertheless, the remaining question is how to formulate and estimate the copula density for dependent random variables. At this point it could still be of arbitrary complexity, leaving the general problems of multivariate random variables, as discussed above, untouched. In fact, there is no general or canonical way to formulate the copula and to assess the correlations among the random variables.

\section{Copula families}

The definition of the parametric form of the copula function or copula density allows to group them into families. Two important classes as used in Sect. 7 are the elliptical and the Archimedian copulas family as given below. Details about other copula families and their selection can be found in Nelsen (2006); Embrechts et al. (2001); Chen and Fan (2005); Genest and Favre (2007); Frees and Valdez (1998); Nelsen (2002). Currently there seems to be no general procedure for copula selection agreed upon. A Bayesian method to select the most probable copula family among a given set is described in Huard et al. (2006).

\subsection{Elliptical copulas}

Elliptical copulas are the copulas of elliptical distributions. For example, the copula of a multivariate normal distribution forms a special case of elliptical copulas. Their main characteristics are that they extend to arbitrary dimensions, but they are comparably rich in parameters: a $m$-dim elliptical copula has at least $m(m-1) / 2$ parameters. Furthermore they are restricted to radial symmetry, what appears to be a strong limitation with respect to tail dependence, i.e. applications like precipitation modeling (see below). Typical examples of elliptical copulas are the normal or Gaussian copula and the Student-t copula.

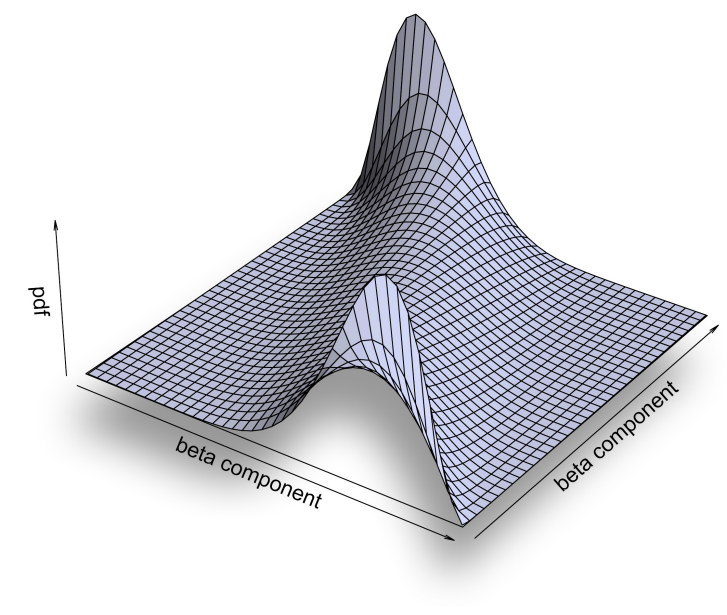

Fig. 2. Example of a bivariate probability density function for a random vector with two Beta distributed vector components with beta parameters $(0.5,0.5)$ and $(2.0,2.0)$ respectively. The dependence among the components is and given by a normal copula with correlation -0.85 .

\subsubsection{Normal copulas}

Normal or Gaussian copulas form a special case of the copula approach, but can also be derived by a simple back and forth transformation of the random variables to the multivariate standard normal distribution. Let $\boldsymbol{X}$ again be a random vector with components $X_{1}, \ldots, X_{m}$ and known marginal cumulative distribution functions $F_{X_{1}}, \ldots, F_{X_{m}}$. By using $U_{j}=F_{X_{j}}\left(X_{j}\right) \sim \mathcal{U}(0,1)$ each random vector component can be transformed to a standard normally distributed random variable

$Z_{j}=F_{\mathcal{N}(0,1)}^{-1}\left(F_{X_{j}}\left(X_{j}\right)\right) \sim \mathcal{N}(0,1)$,

where $j=1, \ldots, m$. Analogously to the classical multivariate normal case we assume that $\boldsymbol{Z}=\left(Z_{1}, \ldots, Z_{m}\right)^{T}$ follows a multivariate standard normal distribution $\mathcal{N}(0, \Sigma)$, with the corresponding probability density function $f_{\mathcal{N}(0, \Sigma)}$ and covariance matrix $\Sigma$.

The probability density function of the original random vector, which is the copula function in the terminology above, is then given by

$$
C_{X}\left(u_{1}, \ldots, u_{m}\right)=F_{\mathcal{N}(0, \Sigma)}\left(F_{\mathcal{N}(0,1)}^{-1}\left(u_{1}\right), \ldots, F_{\mathcal{N}(0,1)}^{-1}\left(u_{m}\right)\right) \text {. }
$$

With the definition of the copula density (Eq. 2) the normal copula density therefore reads

$$
\begin{array}{r}
c_{\boldsymbol{X}}\left(u_{1}, \ldots, u_{m}\right)=\frac{\partial}{\partial u_{1}} \cdots \frac{\partial}{\partial u_{m}} \cdot C_{\boldsymbol{X}}\left(u_{1}, \ldots, u_{m}\right) \\
=\frac{f_{\mathcal{N}(0, \Sigma)}\left(F_{\mathcal{N}(0,1)}^{-1}\left(u_{1}\right), \ldots, F_{\mathcal{N}(0,1)}^{-1}\left(u_{m}\right)\right)}{\prod_{j=1}^{m}\left(f_{\mathcal{N}(0,1)}\left(F_{\mathcal{N}(0,1)}^{-1}\left(u_{m}\right)\right)\right)} .
\end{array}
$$



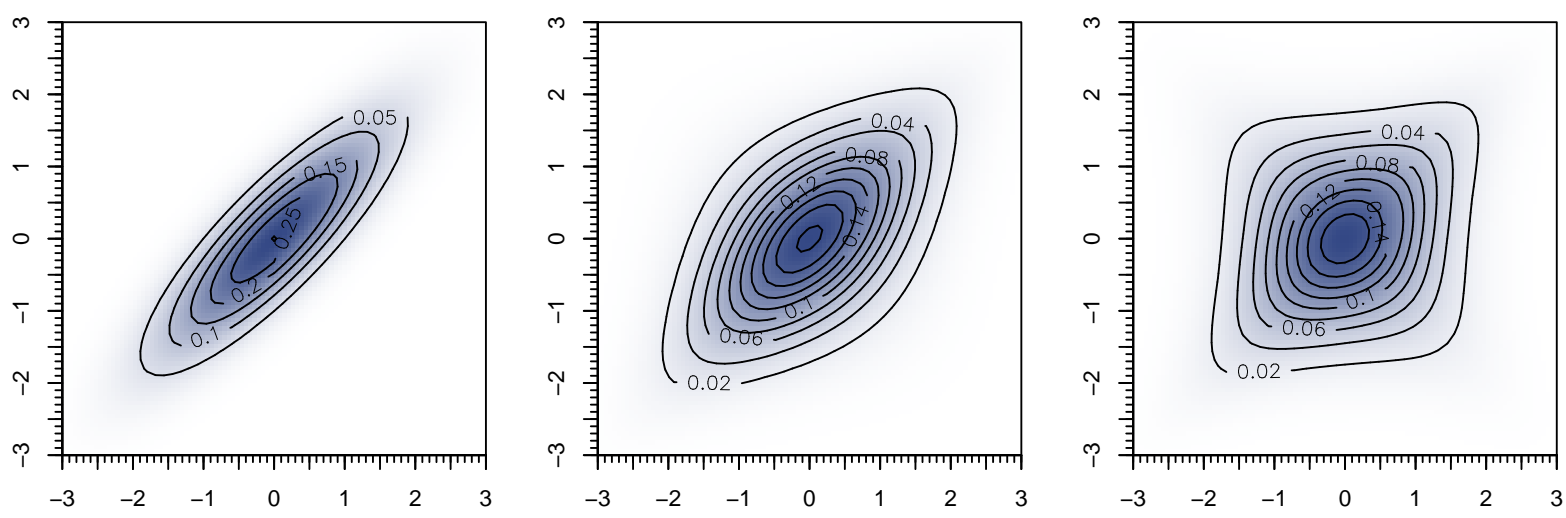

Fig. 3. Probability density functions of different $\mathrm{t}$-copulas with $\rho=0.85$ and $\nu=\infty, 5,2.5$ respectively (from left to right). For better comparability, the marginal distributions are chosen to be standard normal.

Figure 1 shows an example of a probability density function of a two-dimensional random vector with a normally and a Gamma distributed component, Fig. 2 an example for Beta distributed components in order to show the capability of describing bounded distributions.

The normal copula is applicable to many real world problems and the estimation procedures are quality controllable as they are well known. However, it has its limitations, since - analogously to the multivariate normal distribution - it assumes linear relationships among the transformed random variables with fixed and comparably small dependence for large deviations. Further information is given in e.g. Embrechts et al. (2001); Pitt et al. (2006); Renard and Lang (2007); Clemen and Reilly (1999).

\subsubsection{Student's t-copulas}

The t-copula is defined analogously to the Gaussian copula by using a multivariate extension of the t-distribution,

$C_{X}\left(u_{1}, \ldots, u_{m}\right)=F_{t(v, \Sigma)}\left(F_{t(v)}^{-1}\left(u_{1}\right), \ldots, F_{t(v)}^{-1}\left(u_{m}\right)\right)$,

where $F_{t(v)}$ is an univariate t-distribution with $v$ degrees of freedom and positive-definite dispersion or scatter matrix $\Sigma$ leading to a number of $1+m(m-1) / 2$ parameters. For the following applications we refer to the definition by Demarta and McNeil (2005), but note that there are multiple definitions of a multivariate t-distribution and hence of t-copulas (e.g. Shaw and Lee, 2007). Figure 3 shows three bivariate examples for a correlation coefficient of $\rho=0.85$. The degrees of freedom are chosen to be $\nu=\infty$, in which case the t-copula equals a multivariate normal distribution, $v=5$, and $\nu=2.5$, respectively. For better comparison with the multivariate normal distribution the examples are given for standard normal marginals. The reason for this kind of visualization is that the copula function itself is only meaningful w.r.t. the given marginal distributions as pointed out by Mikosch (2006a).
The t-copula allows for some flexibility in covariance structure and tail dependence. Tail dependence can be understood as the conditional probability of observing an extreme in one component given the other being in an extreme state. The corresponding coefficient of tail dependence as introduced below provides an asymptotic measure and is a copula property which is invariant under strictly increasing transformations (e.g. Frahm et al., 2005; Coles et al., 1999; Heffernan, 2000). After Embrechts et al. (2001) the upper tail dependence of a bivariate $t$-copula is given by

$$
\begin{aligned}
\lambda_{\text {up }} & =\lim _{u \nearrow 1} \mathrm{P}\left(X_{1}>F_{X_{1}}^{-1}(u) \mid X_{2}>F_{X_{2}}^{-1}(u)\right) \\
& =2 \lim _{u \nearrow 1} \mathrm{P}\left(U_{1}>u \mid U_{2}=u\right) \\
& =2 F_{t(v+1)}\left(-\sqrt{(v+1) \frac{1-\rho}{1+\rho}}\right)
\end{aligned}
$$

with $\rho=\Sigma_{12} /\left(\Sigma_{11} \Sigma_{22}\right)$ where the second equality follows from the fact that the bivariate t-copula is an exchangeable copula, i.e. $C_{\boldsymbol{X}}\left(u_{1}, u_{2}\right)=C_{\boldsymbol{X}}\left(u_{2}, u_{1}\right)$. Because of the radial symmetry, the lower tail dependence equals the upper tail dependence. As to be expected, it is increasing in $\rho$ and decreasing in $v$. For the examples in Fig. 3 follows $\lambda_{\text {up }}=0$, 0.51 , and 0.63 , respectively. So, the motivation to introduce this framework becomes even clearer: As a special case, the Gaussian copula with $\rho<1$ has in fact zero tail dependence. Therefore, other copulas might be more appropriate for various applications like asymmetric distributions and/or especially large deviations.

Nevertheless, it should be noted that $\lambda_{\text {up/down }}$ as given above is not the ultimate measurement of the tail behavior, since it only describes the asymptotic behavior for $u \nearrow 1$, but not the rate of convergence. For example the Gaussian copula has a slow convergence compared to the Frank copula as shown below (cf. Figs. 3 and 4). Furthermore, the rate of convergence is dependent on the marginal transformation, 

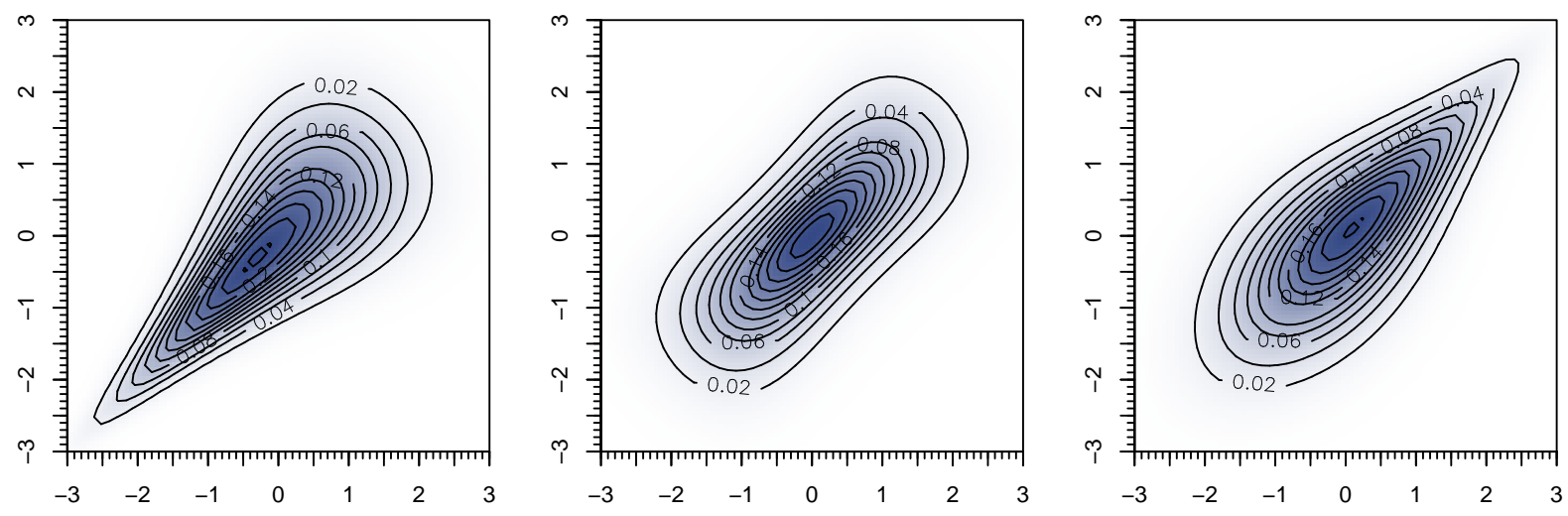

Fig. 4. Probability density functions as given in Fig. 3 but for the Archimendean family: Clayton $\theta_{\mathbf{C}}=2$, Frank $\theta_{\mathrm{F}}=5.736$, and Gumbel $\theta_{\mathrm{G}}=2$ (from let to right).

while $\lambda_{\text {up/down }}$ is not. So it is possible to construct distributions with high correlations for large deviations based on a copula with zero tail dependence. More details on t-copulas can be found in e.g. Kotz et al. (2000).

\subsection{Archimedian copulas}

The copula family introduced above are derived from elliptical distributions using Sklar's theorem. The main drawbacks are therefore that they are restricted to have radial symmetry and do not necessarily exist in closed form expressions. As shown below, practical applications often require different upper and lower tail behavior. A class of copulas that allows for a wider variety of dependence structures is given by the Archimedean copulas,

$C_{\boldsymbol{X}}\left(u_{1}, \ldots, u_{m}\right)=\phi^{-1}\left(\phi\left(u_{1}\right)+\cdots+\phi\left(u_{m}\right)\right)$

with the definitions from above and the function $\phi$ which is called the generator of the copula (Embrechts et al., 2001; Nelsen, 2006; Nelsen et al., 2002). While this multidimensional definition formally follows the criteria of a copula under certain properties of $\phi$, higher dimensions are not practical since the margins are exchangeable. Therefore Archimedean copulas are mostly used in the bivariate case. Details about proper multivariate extensions can be found in Embrechts et al. (2001).

The flexibility of Archimedean copulas is given by the generator functions $\phi$. Commonly used examples are the Clayton, the Frank, and the Gumbel copula,

$$
\begin{aligned}
\phi_{\mathrm{C}}(u) & =u^{-\theta_{\mathrm{C}}}-1 & & \text { (Clayton, 1978) } \\
\phi_{\mathrm{F}}(u) & =\log \left(\frac{e^{\theta_{\mathrm{F}}}-1}{e^{\theta_{\mathrm{F}}}-1}\right) & & (\text { Frank, 1979) } \\
\phi_{\mathrm{G}}(u) & =(-\log u)^{\theta_{\mathrm{G}}} & & \text { (Gumbel, 1960) }
\end{aligned}
$$

As shown in Fig. 4 they allow for different tail behavior. The Clayton copula has lower tail dependence, the Frank copula no tail dependence, and the Gumbel copula only upper tail dependence and is therefore used in Sect. 7. However, since they are not derived from distribution functions as done for the elliptical copulas, the interpretation of their parameters in terms of statistical properties is not straight forward. Nevertheless, the Archimedean copula family plays an important role in fields like risk analysis (Embrechts et al., 2001) and seems to be well applicable to many real world problems in geosciences.

\section{Estimation and GOF tests}

Estimating multivariate distributions using copulas leads to various methods and vast field of literature, where it is hard to keep an overview. Most of the publications focus on the estimation of the copula function. Therefore we commence with an important consideration as given by Mikosch (2006a): The copula concept allows to estimate the marginal distributions and the copula separately, but it is often overlooked that the aim is to fit the whole multivariate distribution. This includes copula and marginals. We introduce three main concepts:

1. An intuitive approach to fit a multivariate distribution is the classical maximum likelihood (ML) estimation of the full multivariate distribution. With a given parametric description of the copula and the marginal distributions, the ML estimate is given by maximizing the log-likelihood function, as follows easily from Eq. (3). Depending on the marginal distribution class and copula family the optimization is only feasible numerically. The main problem is that the numerical complexity of the optimization problem is comparably high: e.g. $\mathrm{O}\left(2 \mathrm{~m}+\mathrm{m}^{2}\right)$ for an $m$-dimensional random vector with two-parameter marginal distributions and an elliptical copula, which is especially relevant since one cannot 
use efficient, predefined estimators for marginal distributions and copula parameters. For this reason it is less used in practice. Nevertheless, it should be noted that the ML estimation of the full distribution is the recommended approach when not dealing with high dimensional random vectors. It is the only way to provide consistent estimates of all parameters and it allows for most control over the fitting process including goodness-offit (GOF) tests.

2. The most commonly used approach is a two-stage estimation process (e.g. Shih and Louis, 1995), which is also called inference functions for margins (IFM). In the first step, the parameters of the margins are estimated as in the univariate case and plugged into the joint probability density, Eq. (3). In the second step, the corresponding likelihood is maximized in order to determine the copula parameters. Since each estimation task has a comparably low number of parameters, it is computationally more efficient than the classical MLE. Furthermore it allows to use predefined estimators for existing classes of marginal distributions and copula families (e.g. Fredricks and Nelsen, 2007). Although the IFM is a convenient approach, a major drawback is often overlooked (Mikosch, 2006a): It does not take into account, that the copula is determined by the parametric models for the margins, and is therefore dependent on their estimation. Hence it is unclear if the IFM provides an efficient estimator in a statistical sense. Nevertheless, it can been shown that the IFM estimator is consistent and asymptotically normal under regularity conditions (Palaro and Hotta, 2006, and references therein).

3. A known alternative is the canonical maximum likelihood (CML) method. It avoids the problem of finding and estimating appropriate parametric models for the margins by using the empirical CDF of each margin instead. This allows for consistent estimates of the copula parameters and their standard errors, but it clearly overrates the theoretical value of the copula itself. It should be noted, that the copula alone does not provide any information about the multivariate distribution without knowing the marginal distributions. Details can be found in Mikosch (2006a) and discussion.

Goodness-of-fit (GOF) tests for copulas relate to the general difficulty of testing multivariate probability densities or distribution functions. While the univariate tests are wellknown, there is less of a common methodology in the multivariate case. Especially copula specific tests are just emerging (Genest et al., 2006).

A simple approach to multivariate GOF tests are multidimensional $\chi^{2}$-tests as given in Fermanian (2005), but they come along with the typical problems of binned approaches. They are sensitive to the selection of bins and there is no optimal choice for the bin width. Furthermore, they are of- ten not feasible for higher dimensional problems since chisquare tests require a sufficient sample size in order for the chi-square approximation to be valid. We therefore focus on alternatives to binned tests.

More efficient GOF tests for copulas are based on dimension reduction approaches using the probability integral transform (PIT), i.e. projecting the multivariate problem to a univariate problem. The PIT transforms an arbitrary multivariate random vector $\boldsymbol{X}$ with components $X_{1}, \ldots, X_{m}$ and known marginal cumulative distribution functions $F_{X_{1}}, \ldots, F_{X_{m}}$ to a set of independent, uniform variables. The PIT of $\boldsymbol{X}$ is defined as

$$
\begin{aligned}
T_{1}\left(X_{1}\right) & =F_{X_{1}}\left(X_{1}\right) \\
T_{2}\left(X_{2}\right) & =F_{X_{2} \mid X_{1}}\left(X_{2} \mid X_{1}\right) \\
& \vdots \\
T_{m}\left(X_{m}\right) & =F_{X_{m} \mid X_{1}, \ldots, X_{m-1}}\left(X_{m} \mid X_{1}, \ldots, X_{m-1}\right) .
\end{aligned}
$$

Under the null hypothesis $\mathcal{H}_{0}$ that $\boldsymbol{X}$ comes from the specified multivariate model, the random variables $Z_{j}^{*}=T_{j}\left(X_{j}\right)$ are then uniformly and independently distributed on $\mathcal{U}(0,1)$. According to Eq. (1) they can be derived via

$$
\begin{aligned}
Z_{j}^{*} & =C_{X_{j} \mid X_{1}, \ldots, X_{j-1}}\left(u_{j} \mid u_{1}, \ldots, u_{j-1}\right) \\
& =\frac{\partial^{j-1} C_{j}\left(u_{1}, \ldots, u_{j}\right)}{\partial u_{1} \cdots \partial u_{j-1}} / \frac{\partial^{j-1} C_{j-1}\left(u_{1}, \ldots, u_{j-1}\right)}{\partial u_{1} \cdots \partial u_{j-1}}
\end{aligned}
$$

with $C_{j}\left(u_{1}, \ldots, u_{j}\right)=C_{\boldsymbol{X}}\left(u_{1}, \ldots, u_{j}, 1, \ldots, 1\right)$.

In the approach proposed by (Breymann et al., 2003) the PIT is performed by defining a univariate random vector

$$
Y^{*}=\sum_{j=1}^{m} F_{\mathcal{N}(0,1)}^{-1}\left(Z_{j}^{*}\right) .
$$

Under $\mathcal{H}_{0}$ it follows a $\chi^{2}$-distribution with $m$ degrees of freedom, so that $W^{*}=F_{\chi_{m}^{2}}\left(Y^{*}\right)$ should be a univariate random variable from $\mathcal{U}(0,1)$. In this way, the problem has been reduced to a univariate problem, which can be evaluated by classical univariate test statistics (Aslan and Zech, 2002). E.g. Breymann et al. (2003) use the Anderson-Darling statistic to carry out the test. While this approach is straight forward and computationally efficient, it has its disadvantages (Berg and Bakken, 2006). Firstly, the PIT depends on the permutation of the random vector components which is a minor problem as long as the permutation is chosen randomly. Secondly, the dimension reduction through $Y^{*}$ causes an inconsistency, so that the test statistic is not strictly increasing for every deviation from $\mathcal{H}_{0}$.

In order to deal with the problem (Berg and Bakken, 2005) propose alternatives to the aforementioned approach. They also provide a detailed comparison of different goodnessof-fit tests based on the PIT as well as the full multivariate approaches by Panchenko (2005). Finally, it should be 
noted that there is currently no universal method for copula goodness-of-fit tests. For further reading we refer to e.g. Genest et al. (2006); Fermanian (2005).

\section{Multivariate extremes}

\subsection{Extreme value theorie}

Extreme value theory is based on a limit law that was first described by Fisher and Tippett (1928) and proven by Gnedenko (1943). It has since then seen great progress (Coles, 2001; Beirlant et al., 2004; Embrechts et al., 2003; Resnick, 1987). In geoscience, extreme value theory has found a wide range of application particularly in hydrology (Gumbel, 1958; Katz, 2002) and more recently also in meteorology and climate (Frei and Schär, 2001; Katz, 2002; Kharin and Zwiers, 2005; Zwiers and Kharin, 1998).

Let us define a maximum $M^{(n)}$ of a finite sequence of length $n$ of identically and independently distributed (i.i.d.) random variables $Y_{i}$ as $M^{(n)}=\max \left(Y_{1}, \ldots, Y_{n}\right)$. The FisherTippett theorem says that there exists a sequence of constants $a^{(n)}>0$ and $b^{(n)}$ such that under very general conditions the distribution function of $\frac{M^{(n)}-b^{(n)}}{a^{(n)}}$ converges to one of the three types (Gumbel, Weibull, or Fréchet) of extreme value distributions.

$\lim _{n \rightarrow \infty} \mathrm{P}\left(\frac{M^{(n)}-b^{(n)}}{a^{(n)}} \leq x\right)=G(x)$

The three types are merged to the generalized extreme value (GEV) distribution. This theorem leads to the limiting distribution for sample maxima (and minima). The GEV distribution reads

$G(x)=\left\{\begin{array}{ll}\exp \left(-\left(1+\xi \frac{x-\mu}{\sigma}\right)^{-1 / \xi}, 1+\xi \frac{x-\mu}{\sigma}>0,\right. & \xi \neq 0 \\ \exp \left(-\exp \left(-\frac{y-\mu}{\sigma}\right)\right), & \xi=0\end{array}\right.$,

and represents the limiting distribution for sample maxima (and minima). The GEV is defined by three parameters, the location $\mu$, the scale $\sigma$ and the shape $\xi$ parameter. The sign of the shape parameter determines the type of extreme value distribution, with $\xi=0$ defines the Gumbel, $\xi>0$ the Fréchet, and $\xi<0$ the Weibull type. If the maxima of a process $Y$ follow a GEV, then it is said to be in the domain of attraction of an extreme value distribution. This is the general condition under which EVT applies.

\subsection{Extreme value copulas}

Unlike in univariate extreme value theory, the class of possible limiting distribution functions of extreme values cannot be captured by a finite-dimensional parametric family of functions. The study of multivariate extremes splits into the description of the marginal distribution and the dependence structure. In order to characterize the limit behavior of multivariate extremes, it has been shown, that weak convergence of the multivariate distribution is equivalent to weak convergence of the marginals as well as the copula function, provided that the marginals are continuous (Beirlant et al., 2004). This is the reason why the copula approach is very popular in modeling multivariate extremes (e.g. Renard and Lang, 2007). In contrast to the copula concept, in multivariate extreme value theory a particularly useful choice is to work with standard Fréchet margins.

Multivariate extreme value theory defines limiting distribution functions for the maximum of a sequence of multivariate random variates $\boldsymbol{Y}_{i}$ of dimension $m$. The multivariate sample maximum is defined by marginal ordering, where the maximum of a sequence of multivariate variates is the component-wise maximum

$$
\boldsymbol{M}^{(n)}=\left(\max \left(Y_{11}, \ldots, Y_{m 1}\right), \ldots, \max \left(Y_{1 n}, \ldots, Y_{m n}\right)\right)^{T}
$$

with sequence size $n$ and dimension $m$. Under certain conditions the distribution of the vector of normalized maxima follows a non-degenerate multivariate extreme value distribution $G_{X}(x)$ The marginal distributions $G_{X_{j}}\left(x_{j}\right)$ in turn follow a univariate generalized extreme value distribution. In extreme value theory it is convenient to standardize the margins to standard Fréchet. If $X_{j}$ follows $G_{X_{j}}$ with parameters $\mu, \sigma$, and $\xi$,

$Z_{j}=-\frac{1}{\log \left(G_{X_{j}}\left(X_{j}\right)\right)}=\left(1+\xi\left(\frac{X_{j}-\mu}{\sigma}\right)\right)^{1 / \xi}$

follows a standard Fréchet distribution with $\mathrm{P}\left(Z_{j} \leq z\right)=\exp (-1 / z)$. Then, the multivariate extreme value distribution is of the form

$$
\begin{aligned}
G_{\boldsymbol{X}}(\boldsymbol{x}) & =G_{\boldsymbol{Z}}^{*}(z) \\
& =\exp \left(-V^{*}\left(z_{1}, \ldots, z_{m}\right)\right) \\
& =\exp \left(-V^{*}\left(\frac{1}{-\log \left(G_{X_{1}}\left(x_{1}\right)\right)}, \ldots, \frac{1}{-\log \left(G_{X_{m}}\left(x_{m}\right)\right)}\right)\right),
\end{aligned}
$$

where $V^{*}$ is denoted as dependence function. In the bivariate case, the dependence function can be expressed as

$V\left(z_{1}, z_{2}\right)=2 \int_{0}^{1} \max \left(\frac{\omega}{z_{1}}, \frac{1-\omega}{z_{2}}\right) d H(\omega)$.

The spectral measure $H(\omega)$ is a distribution function on the interval $[0,1]$ with $\int_{0}^{1} \omega d H(\omega)=1 / 2$. Hence, in the bivariate case the dependence function is determined by a one dimensional spectral measure $H$. However, the spectral measure has no finite-parameter form, it is not necessarily differentiable, and many families of parametric functions satisfy the condition (e.g. logistic family). An important property is that $V$ is homogeneous of order -1 ,

$V(s \boldsymbol{z})=\frac{1}{s} V(\boldsymbol{z})$ for $0<s<\infty$, 

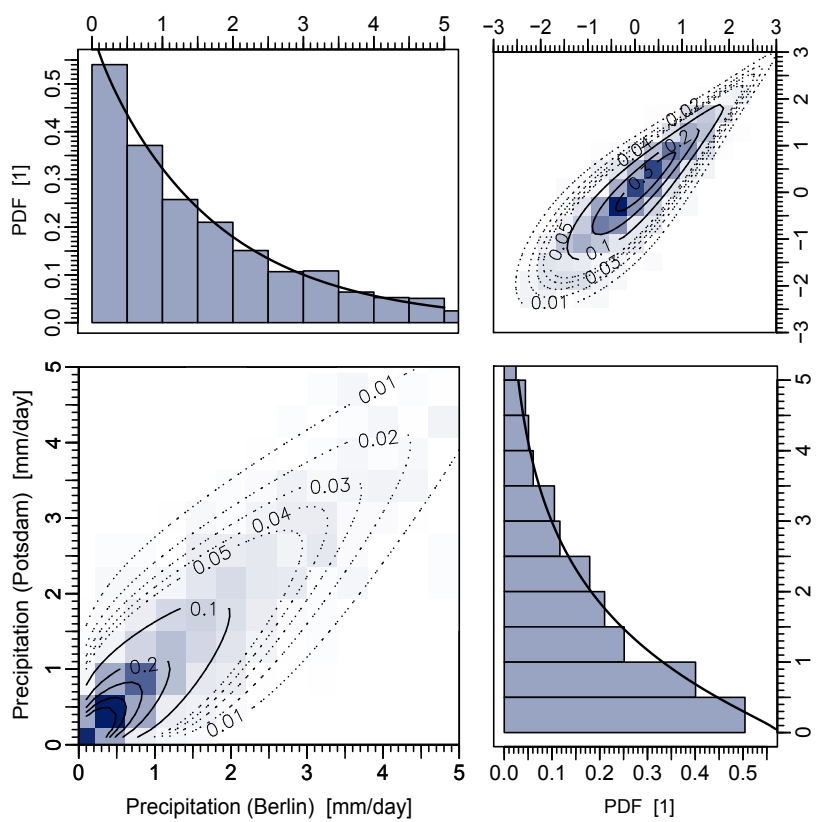

Fig. 5. Bivariate distribution of average daily precipitation at station Berlin and Potsdam (see text). The bottom left plot shows the estimated probability density functionand the top right plot the copula density analogously to Figs. 3 and 4. The parameter estimates (and standard errors) of the gamma distributions and the Gumbel copula are are $\hat{\alpha}_{\text {Berlin }}=0.96(0.033), \hat{\beta}_{\text {Berlin }}=0.56(0.024), \hat{\alpha}_{\text {Potsdam }}=1.00$ (0.036), $\hat{\beta}_{\text {Potsdam }}=0.61(0.034)$, and $\hat{\theta}_{\mathrm{G}}=3.06(0.096)$.

which follows from Eq. (6) and equally holds for the multivariate case. This condition assures that $G_{X}(x)$ is maxstable, as $G_{X}^{n}(\boldsymbol{x})=G_{\boldsymbol{X}}\left(n^{-1} \boldsymbol{x}\right)$, which guarantees, that the maximum of a sample of $\boldsymbol{x}$ drawn from $G_{X}$ again follows a multivariate generalized extreme value distribution.

An alternative description of a multivariate extreme value distribution is through its copula. The copula of a multivariate extreme value distribution $G_{\boldsymbol{X}}$ with marginals $G_{X_{j}}\left(x_{j}\right)$ is given by

$G_{\boldsymbol{X}}(\boldsymbol{x})=C_{\boldsymbol{X}}\left(G_{X_{1}}\left(x_{1}\right), \ldots, G_{X_{m}}\left(x_{m}\right)\right)$

Because the copula is defined on uniform margins, it can be written in form of the dependence function $V^{*}$ as

$C_{\boldsymbol{X}}(\boldsymbol{u})=\exp \left(-V^{*}\left(-\frac{1}{\log \left(u_{1}\right)}, \ldots, \frac{1}{\log \left(u_{m}\right)}\right)\right)$

with $\boldsymbol{u} \in[0,1]^{m}$. A necessary condition $C_{\boldsymbol{X}}$ must satisfy to be a copula of a multivariate extreme value distribution is the homogeneity condition

$C_{\boldsymbol{X}}^{s}(\boldsymbol{u})=C_{\boldsymbol{X}}\left(u_{1}^{s}, \ldots, u_{m}^{s}\right)$.

This stability relation follows from Eq. (7). Conversely, any copula that satisfies Eq. (8) is a copula of a multivariate extreme value distribution. As for the multivariate generalized extreme value distribution, condition (8) ensures that the extreme value copula is max-stable.
Pickands (1981) proposed a procedure for constructing an extreme value copula. In the bivariate case, the Pickands dependence function $A$ determines an extreme value copula (Nelsen, 2006) with

$C_{\boldsymbol{X}}\left(u_{1}, u_{2}\right)=\exp \left(\log \left(u_{1} u_{2}\right) \cdot A\left(\frac{\log \left(u_{2}\right)}{\log \left(u_{1} u_{2}\right)}\right)\right)$

$A$ is a mapping of the interval $[0,1] \rightarrow[1 / 2,1]$ and must satisfy certain conditions: (a) $A(0)=A(1)=1$, (b) $\max (t, 1-t) \leq A(t) \leq 1$, and (c) $A$ is convex.

Since this section gives a short conceptional overview of multivariate extreme value theory, in order to relate it to the copula approach for extreme value distributions, the reader is referred to Coles (2001); Coles and Pauli (2002); Beirlant et al. (2004) and references therein for further reading.

Much work in multivariate extreme value theory has centered around describing the dependence of extreme observations de Haan and Resnick (1993); Coles and Tawn (1996); Coles et al. (1999); Schlather and Tawn (2003); Heffernan and Tawn (2004); Mendes et al. (2006). Recent studies investigate the spatial dependencies of precipitation extremes Vannitsem (2007); Vrac and Naveau (2007); Vrac et al. (2007); Cooley et al. (2007), where the idea is to combine geostatistics with multivariate extreme value theory.

\section{Applications}

In order to show the application of the copula framework, three typical situations of bivariate random variables are considered: (1) the same meteorological parameter at different locations, (2) different meteorological parameters at the same location, and (3) bivariate extremes, as far as they can be expressed in the copula framework.

For the first two cases, we selected daily observations during the winter months (DJF) taken from the Global Historical Climatology Network (GHCN) that passed the internal quality control. They are split into five-day chunks, the typical life time of synoptical disturbances, with two-days gaps in order to reduce the autocorrelation, which is then between 0.07 and 0.15 . Since we do not explicitly account for temporal dependence within these examples, we would cause an under estimation of the standard errors otherwise.

In the first application a bivariate random vector is composed of five-day averages of precipitation measurements at two stations in Germany which are at small distance from each other: WMO station 10389 Berlin $(52.45 \mathrm{~N}, 13.30 \mathrm{E})$ at $55 \mathrm{~m}$ MSL and WMO station 10379 Potsdam $(52.38 \mathrm{~N}, 13.07 \mathrm{E})$ at $81 \mathrm{~m}$ MSL. Common observations are available between 1893 and 2005 leading to a sample size of 1218 elements. Only non-zero precipitation amounts at both stations are considered, which leads to a slightly different marginal distribution in the second example. Furthermore, rain gauges measure in $0.2 \mathrm{~mm}$ steps leading to an artificial discretization, especially in lower precip- 
itation amounts. To overcome this problem a random noise drawn from $\mathcal{U}(-0.1,0.1)$ has been added, which is to small to affect the parameter estimates, but removes the round offeffect. Figure 5 shows the corresponding fit of a multivariate distribution with Gamma distributed marginals and a Gumbel copula. The parameter estimates are based on the full maximum likelihood method (Sect. 5), which allows independent error estimates for the margins and the copula parameter. A goodness of fit test using the PIT and the Anderson-Darling test gives a p-value of 0.35 .

The transformed random vector components, in the example chosen, are strongly correlated with only upper tail dependence. Figure 5 (top right) indicates why e.g. an elliptical copula would not be sufficient to describe the dependence. The asymmetric tail behavior also excludes regular correlation coefficients as a proper measurement of dependence. It has two reasons. The copula strongly depends on the selection of the marginal distribution and in this case, the transformations of the margins are highly nonlinear, especially for small precipitation values $\left(X_{j} \rightarrow 0 \Rightarrow Z_{j} \rightarrow-\infty\right)$. Furthermore, large precipitation amounts are indeed well correlated on the respective averaging period. This illustrates that the copula does not provide a practical measurement of tail behavior of the original random variables, but upper/lower tail dependence of the transformed variables has to be considered when fitting a copula model. For example, fitting the same data to Gaussian copula would diminish the GOF test to a p-value of 0.014

It should also be mentioned, that a bivariate Gamma distribution could be used as alternative. Nevertheless, since there are several definitions of bivariate Gamma distributions (Izawa, Moran, Smith-Adelfang-Tubbs, ...) and some of them are even identical to the copula concept, e.g. the FarlieGumbel-Morgenstern model we refer to a review of bivariate gamma distributions (Yue et al., 2001).

In the second application a bivariate random vector is composed of five-days averages of precipitation and five-days minima of temperature at the station Berlin. The marginal distributions are described by a Gamma distribution for precipitation and Gumbel distribution for the temperature minimum. The aim is to test if a simple statistical model based on the copula approach is capable of describing the phenomenon that cold periods are accompanied by small precipitation amounts. In opposition to the application above the dependence between the transformed random variables is small. Furthermore, it appears to be symmetric with small or no tail dependence, which indicates to use a Gaussian copula (Fig. 6). Estimation and Goodness-of-fit test are performed as above and lead to a p-value of 0.38 . Nevertheless, it is well known that selecting the Gamma distribution as a parametric description for precipitation amounts is problematic when focusing on extreme precipitation events (e.g. Vrac and Naveau, 2007).
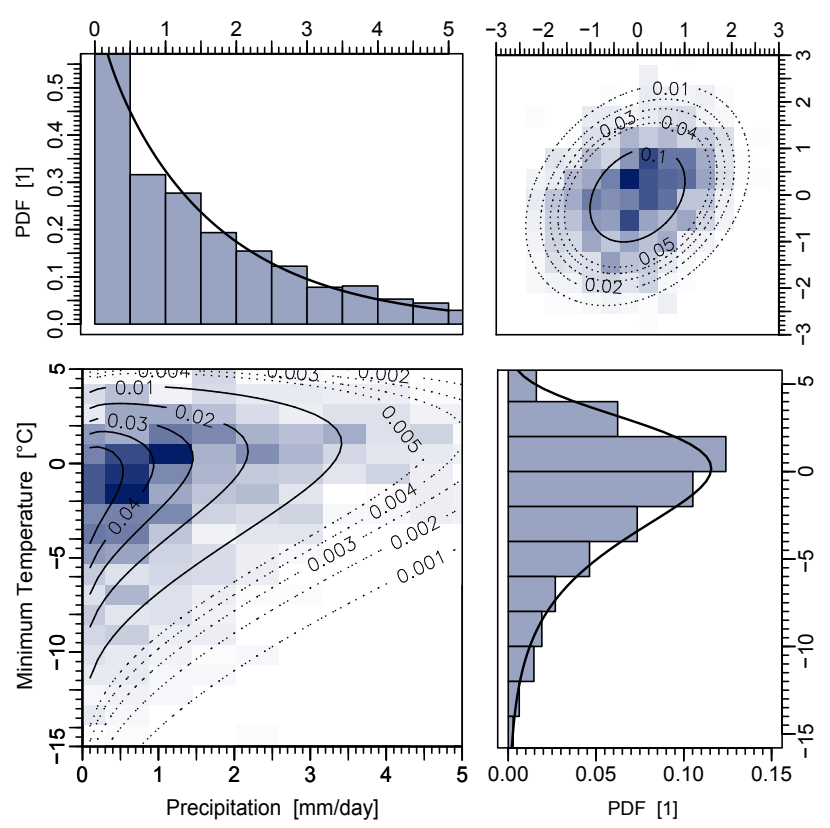

Fig. 6. Bivariate distribution of average daily precipitation and minimum temperature at station Berlin (see text and Fig. 5). The parameter estimates (and standard errors) of the gamma distribution, the Gumbel distribution, and the normal copula are $\hat{\alpha}_{\text {Precip }}=0.93(0.030), \hat{\beta}_{\text {Precip }}=0.57(0.024), \hat{\mu}_{\text {Tmin }}=-0.18$ $(0.088), \hat{\beta}_{\mathrm{Tmin}}=3.18(0.065)$, and $\hat{\rho}_{12}=0.29(0.023)$.

For the third application daily wind maxima at two closeby locations are considered: WMO station 10382 BerlinTegel $(52.28 \mathrm{~N}, 13.24 \mathrm{E})$ at $36 \mathrm{~m}$ MSL and WMO station 10384 Berlin-Tempelhof $(52.34 \mathrm{~N}, 13.18 \mathrm{E})$ at $49 \mathrm{~m}$ MSL, both taken from the station network of the German weather service DWD. Only fall and winter month (ONDJFM) between 2003 and 2007 are taken into account, leading to approx. 1460 observations. The wind measurements are stored in steps of $1 \mathrm{~m} / \mathrm{s}$, so analogously to the examples above, a random noise drawn from $\mathcal{U}(-0.5,0.5)$ has been added in order to avoid an artificial discretisations. Since we are given block maxima, the marginal distributions are described by a generalized extreme value distribution (GEV) with location parameters $\mu$, scale $\sigma$, and shape $\xi$. Their dependence is described by a Gumbel copula which is also an extreme value copula, since it satisfies Eq. (8). Figure 7 shows the corresponding fit of the full multivariate distribution. As to be expected for daily wind maxima the estimates of the shape parameter are close to zero, so they belong to the Gumbel class indicating that they are neither heavy-tailed nor bounded. The covariance structure is well captured as illustrated in the upper right panel of Fig. 7. As before, a goodness of fit test using the PIT and the Anderson-Darling test gives a $\mathrm{p}$-value of 0.84 . Nonetheless, the problem is often infeasible for a higher number of dimensions or other parameters due 

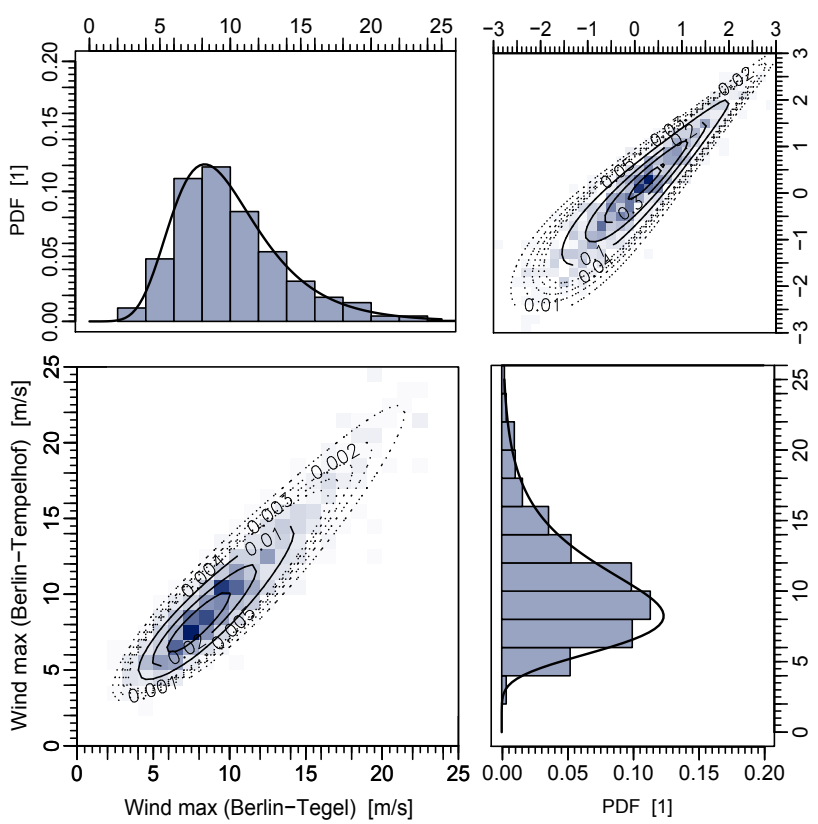

Fig. 7. Bivariate distribution of daily wind maxima at station BerlinTegel and Berlin-Tempelhof (see text and Fig. 5). The parameter estimates (and standard errors) of the GEV distribution, and the Gumbel copula are $\hat{\mu}_{\mathrm{Teg}}=8.24(0.12), \hat{\sigma}_{\mathrm{Teg}}=3.05(0.09), \hat{\xi}_{\mathrm{Teg}}=0.021$ (0.026), $\hat{\mu}_{\mathrm{Tem}}=8.41(0.088), \hat{\sigma}_{\mathrm{Tem}}=2.98 \quad(0.09), \hat{\xi}_{\mathrm{Tem}}=0.059$ (0.027), and $\hat{\theta}_{\mathrm{G}}=4.09(0.12)$.

to a low number of observations compared to high-resolution wind measurements. For related and more detailed applications concerning multivariate extremes see e.g. de Haan and de Ronde (1998); Michele and Salvadori (2003); Coles and Walshaw (1994); Palutikof et al. (1999).

The examples above demonstrate the applicability of the copula approach to meteorological parameters and one can think of numerous further applications. A few of them are given e.g. in the in the field of paleoclimatology (Neumann et al., 2007; Schölzel, 2006; Kühl et al., 2007). Furthermore, the copula concept allows to introduce covariates so that it might gain interest in the the vast field of downscaling.

\section{Conclusions}

The theme of this paper is how recent developments in the statistics of multivariate random variables can be applied to improve meteorological or climatological applications. The copula approach can be seen as a simple and straight forward method to find parametric descriptions of multivariate non-normally distributed random variables. Although it is a fast growing field in statistics, it is still less known in climate research, where often non-normally distributed random vari- ables like precipitation, wind speed, cloud cover, humidity, etc. are in involved.

The strength of this approach is to catch various covariance structures while keeping proper parametric descriptions of the margins. Compared to mixture models, which could be claimed to be an alternative, the copula concept comes along with two major advantages. Mixture models allow only approximative descriptions of the univariate marginal distribution functions and even simple covariance structures might end up in a large number of mixture components in order to provide sufficiently good fits - especially under the aspect of tail dependence. Since concept behind mixture modeling assumes different populations it is rather an enhancement than an alternative. In fact, mixture models and copulas can be combined as shown in Diday and Vrac (2005); Vrac et al. (2005); Hu (2006). Speaking of alternatives, one might also think of a classical way to avoid the problem by transforming the margins and using a standard multivariate normal distribution, but it should be pointed out that this is only a specific case of the larger framework presented here and the examples show that linear correlation coefficients are not a proper measurement of dependence outside the world of elliptical distributions.

The limitations of the copula approach are given in an extensive and interesting discussion, initiated by Mikosch (2006a). The discussion as well as the rejoinder (Mikosch, 2006b) are recommended for further reading as they point out typical problems and misunderstandings:

There is no general procedure for selecting the copula class. As for any kind of statistical model, a satisfying answer does not yet exist, even in the univariate case, but the copula concept allows to use the same strategies as in any other modeling approach, i.e., physical background information, cross-validation, etc. (Genest and Favre, 2007). In any case, if available, one should use a multivariate distribution which is suited to the problem. Furthermore the theoretical value of the copula must not be exaggerated. The copula of a distribution is determined by the marginal distribution which also dictates the rate of convergence in the tail dependence, as perfectly seen in Sect. 7. Direct interpretations of the copula function alone do not provide insight into the stochastical nature of the observed process. There is no dependence separately from the marginal distributions.

Finally, it is clear that the copula approach does not solve the problem of dimensionality. Although copulas allow for various kinds of dependence structure, the problem of finding parametric distributions for high dimensional random vectors remains complex. Any kind of high dimensional multivariate distribution is either limited in covariance structure or comes along with a high number parameters.

As a conclusion, the copula framework provides a vast field for future applications. Due to the need for multivariate methods, statistical literature on copula modeling has been growing over the last few years - and its connection to the modeling of multivariate extremes increases its popularity. 
After numerous successful applications in risk management, financial research and more recently in hydrology, it is very likely that copulas will have growing impact in the field of meteorology and climate research.

Acknowledgements. This work is part of the PArK project which is supported by the Landesanstalt für Umwelt, Messungen und Naturschutz Baden-Württemberg ( $L U B W$ ). The authors would also like to credit the contributors of the $R$ project.

Edited by: P. Yiou

Reviewed by: M. Kallache and two other anonymous referees

\section{References}

Aslan, B. and Zech, G.: Comparison of different goodness-of-fit tests, ArXiv Mathematics e-prints, math/0207300, 2002.

Bardossy, A. and Li, J.: Geostatistical interpolation using copulas, Water Resour. Res., 44, W07412, doi:10.1029/2007WR006115, 2008.

Beirlant, J., Goegebeur, Y., Segers, J., and Teugels, J.: Statistics of Extremes: Theory and Applications, Wiley, 2004.

Berg, D. and Bakken, H.: A Goodness-of-fit Test for Copulae Based on the Probability Integral Transform, Tech. Rep. 10, Department of Mathematics, University of Oslo, 2005.

Berg, D. and Bakken, H.: Copula Goodness-of-fit Tests: A Comparative Study, http://www.danielberg.no/publications/CopulaGOF. pdf, last access 10 October 2008, 2006.

Breymann, W., Dias, A., and Embrechts, P.: Dependence structures for multivariate high-frequency data in finance, Quant. Financ., 3, 1-14, 2003.

Cario, M. C. and Nelson, B. L.: Modeling and generating random vectors with arbitrary marginal distributions and correlation matrix, Working paper, Department of Industrial Engineering and Management Sciences, Northwestern University, 1997.

Chen, X. and Fan, Y.: Pseudo-likelihood ratio tests for semiparametric multivariate copula model selection, The Canadian Journal of Statistics, 33, 389-414, 2005.

Clayton, D.: A model for association in bivariate life tables and its application in epidemiological studies of familial tendency in chronic disease incidence, Biometrika, 65, 141-152, 1978.

Clemen, R. T. and Reilly, T.: Correlations and Copulas for Decision and Risk Analysis, Health Care Management Science, 45, 208224, 1999.

Coles, S.: An Introduction to Statistical Modeling of Extreme Values, Springer Series in Statistics, Springer, New York, 2001.

Coles, S. and Pauli, F.: Models and inference for uncertainty in extremal dependence, Biometrika, 89, 183-196, 2002.

Coles, S., Heffernan, J., and Tawn, J.: Dependence Measures for Extreme Value Analyses, Extremes, 2, 339-365, doi:10.1023/A:1009963131610, 1999.

Coles, S. G. and Tawn, J. A.: Modelling Extremes of the Areal Rainfall Process, J. R. Stat. Soc., Series B, 58, 329-347, 1996.

Coles, S. G. and Walshaw, D.: Directional Modelling of Extreme Wind Speeds, Appl. Stat., 43, 139-157, 1994.

Cooley, D., Nychka, D., and Naveau, P.: Bayesian Spatial Modeling of Extreme Precipitation Return Levels, J. Am. Stat. Assoc., 102, 824-840, 2007. de Haan, L. and de Ronde, J.: Sea and Wind: Multivariate Extremes at Work, Extremes, 1, 7-45, doi:10.1023/A:1009909800311, 1998.

de Haan, L. and Resnick, S.: Estimating the limit distribution of multivariate extremes, Communications in Statistics - Stochastic Models, 9, 275-309, 1993.

Demarta, S. and McNeil, A.: The t Copula and Related Copulas, Int. Stat. Rev., 73, 111-129, 2005.

Diday, E. and Vrac, M.: Mixture decomposition of distributions by copulas in the symbolic data analysis framework, Journal of Discrete Applied Mathematics, 147, 27-41, 2005.

Eckmann, J.-P. and Ruelle, D.: Ergodic theory of chaos and strange attractors, Rev. Mod. Phys., 57, 617-656, 1985.

Embrechts, P., Lindskog, F., and McNeil, A.: Modelling Dependence with Copulas and Applications to Risk Management, in: Handbook of Heavy Tailed Distributions in Finance, edited by: Rachev, S., 329-384., Elsevier, 2001.

Embrechts, P., Klüppelberg, C., and Mikosch, T.: Modelling Extremal Events for Insurance and Finance, Springer, Berlin, 2003.

Fermanian, J.-D.: Goodness-of-fit tests for copulas, J. Multivariate Anal., 95, 119-152, 2005.

Fisher, R. A. and Tippett, L. H.: On the estimation of the frequency distributions of the largest or smallest member of a sample, Proc. Cambridge Phil. Soc., 24, 180-190, 1928.

Frahm, G., Junker, M., and Schmidt, R.: Estimating the taildependence coefficient: Properties and pitfalls, Insurance: Mathematics and Economics, 37, 80-100, 2005.

Frank, M.: On the simultaneous associativity of $f(x, y)$ and $x+y-f(x, y)$, Aequationes Mathematicae, 19, 194-226, 1979.

Fredricks, G. A. and Nelsen, R. B.: On the relationship between Spearman's rho and Kendall's tau for pairs of continuous random variables, 2007, 137, 2143-2150, 2007.

Frees, E. W. and Valdez, E. A.: Understanding Relationships Using Copulas, North American Actuarial Journal, 2, 1-37, 1998.

Frei, C. and Schär, C.: Detection probability of trends in rare events: Theory and application to heavy precipitation in the Alpine region, J. Climate, 14, 1568-1584, 2001.

Genest, C. and Favre, A.-C.: Everything You Always Wanted to Know about Copula Modeling but Were Afraid to Ask, J. Hydrol. Eng., 12, 347-368, 2007.

Genest, C., Quessy, J.-F., and Millard, B.: Goodness-of-fit Procedures for Copula Models Based on the Probability Integral Transformation, Scand. J. Stat., 33, 337-366, 2006.

Genest, C., Favre, A.-C., Béliveau, J., and Jacques, C.: Metaelliptical copulas and their use in frequency analysis of multivariate hydrological data, Water Resour. Res., 43, W09401, doi:10.1029/2006WR005275, 2007.

Gnedenko, B.: Sur la distribution limite du terme maximum d'une serie aleatoire, Ann. Math., 44, 423-453, 1943.

Gumbel, E.: Bivariate exponential distributions, Journal of the American Statistical Association, 55, 698-707, 1960.

Gumbel, E. J.: Statistics of Extremes, Columbia University Press, 1958.

Haas, C. N.: On Modeling Correlated Random Variables in Risk Assessment, Risk Analysis, 1205-1214, 1999.

Heffernan, J. E.: A Directory of Coefficients of Tail Dependence, Extremes, 3, 279-290, doi:10.1023/A:1011459127975, 2000.

Heffernan, J. E. and Tawn, J. A.: A conditional approach for multivariate extreme values, J. Roy. Stat. Soc. B., 66, 497-546, 2004. 
Hu, L.: Dependence patterns across financial markets: a mixed copula approach, Applied Financial Economics, 16, 717-729, doi:10.1080/09603100500426515, 2006.

Huard, D., Evina, G., and Favre, A.-C.: Bayesian copula selection, Computational Statistics and Data Analysis, 51, 809-822, 2006.

Johnson, N. L.: Bivariate Distributions Based on Simple Translation Systems, Biometrika, 36, 297-304, 1949.

Katz, R. W.: Techniques for estimating uncertainty in climate change scenarios and impact studies, Climte Res., 20, 167-185, 2002.

Kharin, V. and Zwiers, F.: Estimating extremes in transient climate change simulations, J. Climate, 18, 1156-1173, 2005.

Kühl, N., Litt, T., Schölzel, C., and Hense, A.: Eemian and Early Weichselian temperature and precipitation variability in northern Germany, Quaternary Sci. Rev., 26, 3311-3317, doi:10.1016/j.quascirev.2007.10.004, 2007.

Kotz, S., Balakrishnan, N., and Johnson, N. L.: Continuous Multivariate Distributions, Wiley-Interscience, New York, 2nd edn., 2000.

Lorenz, E. N.: The problem of deducing the climate from the governing equations, Tellus, 16, 1-11, 1964.

Marin, J.-M., Mengersen, K. L., and Robert, C.: Bayesian modelling and inference on mixtures of distributions, in: Bayesian Thinking, Modeling and Computation, edited by: Dey, D. and Rao, C., vol. 25 of Handbook of Statistics, Elsevier, 2005.

McLachlan, G. and Peel, D.: Finite Mixture Models, WileyInterscience, 2000.

Mendes, J. M., Turkman, K. F., and Corte-Real, J.: A Bayesian hierarchical model for local precipitation by downscaling large scale atmospheric circulation patterns, Environmetrics, 17, 721738, 2006.

Michele, C. D. and Salvadori, G.: A Generalized Pareto intensityduration model of storm rainfall exploiting 2-Copulas, J. Geophys. Res., 108(D2), 4067, doi:10.1029/2002JD002534, 2003.

Mikosch, T.: Copulas: Tales and facts, Extremes, 9, 3-20, doi:10.1007/s10687-006-0015-x, 2006a.

Mikosch, T.: Copulas: Tales and facts - rejoinder, Extremes, 9, 5562, doi:10.1007/s10687-006-0024-9, 2006b.

Nelsen, R. B.: Concordance and copulas: A survey, in: Distributions with Given Marginals and Statistical Modelling, edited by: Cuadras, C. M., Fortiana, J., and Lallena, J. A. R., 169-178, Kluwer Academic Publishers, Dordrecht, 2002.

Nelsen, R. B.: An Introduction to Copulas, Springer, New York, 2nd edn., 2006.

Nelsen, R. B., Molina, J. J. Q., Lallena, J. A. R., and Flores, M. U.: Multivariate Archimedian quasi-copulas, in: Distributions with Given Marginals and Statistical Modelling, edited by: Cuadras, C. M., Fortiana, J., and Lallena, J. A. R., 179-186, Kluwer Academic Publishers, Dordrecht, 2002.

Neumann, F., Schölzel, C., Litt, T., Hense, A., and Stein, M.: Holocene vegetation and climate history of the northern Golan heights (Near East), Veg. Hist. Archaeobot., 16, 329-346, 2007.

Palaro, H. and Hotta, L.: Using Conditional Copula to Estimate Value at Risk, Journal of Data Science, 4, 93-115, 2006.

Palutikof, J. P., Brabson, B. B., Lister, D. H., and Adcock, S. T.: A review of methods to calculate extreme wind speeds, Meteorol. Appl., 6, 119-132, 1999.
Panchenko, V.: Goodness-of-fit test for copulas, Physica A: Statistical Mechanics and its Applications, 355, 176-182, 2005.

Pickands, J.: Multivariate extreme value distributions, In Bull. Int. Statist. Inst., Proceedings of the 43rd Session of the I.S.I., 859878, 1981.

Pitt, M., Chan, D., and Kohn, R.: Efficient Bayesian inference for Gaussian copula regression models, Biometrika, 93, 537-554, 2006.

Renard, B. and Lang, M.: Use of a Gaussian copula for multivariate extreme value analysis: Some case studies in hydrology, Adv. Water Resour., 30, 897-912, 2007.

Resnick, S.: Extreme values, regular variation, and point processes, Springer-Verlag, New York, 1987.

Schlather, M. and Tawn, J. A.: A dependence measure for multivariate and spatial extreme values: properties and inference, Biometrika, 90, 139-156, 2003.

Schölzel, C.: Palaeoenvironmental transfer functions in a Bayesian framework with application to holocene climate variability in the Near East, vol. 62 of Bonner Meteorologische Abhandlungen, Bonn, 2006.

Shaw, W. and Lee, K.: Bivariate Student t distributions with variable marginal degrees of freedom and independence, J. Multivariate Anal., 99, 1276-1287, doi:10.1016/j.jmva.2007.08.006, 2007.

Shih, J. H. and Louis, T. A.: Inferences on the Association Parameter in Copula Models for Bivariate Survival Data, Biometrics, 51, 1384-1399, 1995.

Storch, H. v. and Zwiers, F. W.: Statistical Analysis in Climate Research, Cambridge University Press, 1999.

Vannitsem, S.: Statistical properties of the temperature maxima in an intermediate order Quasi-Geostrophic model, Tellus A, 59, 80-95, doi:10.1111/j.1600-0870.2006.00206.x, 2007.

Vrac, M. and Naveau, P.: Stochastic downscaling of precipitation: From dry events to heavy rainfalls, Water Resour. Res., 43, W07402, doi:10.1029/2006WR005308, 2007.

Vrac, M., Chédin, A., and Diday, E.: Clustering a Global Field of Atmospheric Profiles by Mixture Decomposition of Copulas, J. Atmos. Ocean. Tech., 22, 1445-1459, 2005.

Vrac, M., Naveau, P., and Drobinski, P.: Modeling pairwise dependences in precipitation intensities, Nonlin. Processes Geophys., 14, 789-797, 2007, http://www.nonlin-processes-geophys.net/14/789/2007/.

Wagner, M. A. F. and Wilson, J. R.: Using bivariate Bezier distributions to model simulation input processes, in: Proceedings of the Winter Simulation Conference, 324-331, Orlando, FL, 1994.

Wilks, D. S.: Statistical Methods in the Atmospheric Sciences, Academic Press, 2nd edn., 2005.

Wilson, J. R.: Modeling Dependencies in Stochastic Simulation Inputs, in: 1997 Winter Simulation Conference, edited by: Andradottir, S., Healy, K. J., Withers, D. H., and Nelson, B. L., Atlanta, Georgia, 1997.

Yue, S., Ouarda, T. B. M. J., and Bobee, B.: A review of bivariate gamma distributions for hydrological application, J. Hydrol., 246, 1-18, 2001.

Zwiers, F. W. and Kharin, V. V.: Changes in the extremes of the climate simulated by CCC GCM2 under $\mathrm{CO}_{2}$ doubling, J. Climate, 11, 2200-2222, 1998. 